\title{
A Robust Solution for PI-based LFC Problem with Communication Delays
}

\author{
Hassan Bevrani* Member \\ Takashi Hiyama* Member
}

\begin{abstract}
This paper addresses a new method to design a PI-based load-frequency control (LFC) with communication delays. First the LFC problem is reduced to a static output feedback control synthesis for a multiple delays system, and then the control parameters are easily carried out using robust $H_{\infty}$ control technique. A 3-control area power system example is given to illustrate the proposed control methodology and the results are compared with the $H_{\infty}$-based delay-less control system design.
\end{abstract}

Keywords: $H_{\infty}$ control, LFC, time-delay systems, static output feedback control, LMI approach, robust performance

\section{Introduction}

Currently in many countries, power electric systems are restructured. Operating the power system in a new environment will certainly be more complex than in the past due to the considerable degree of interconnection, and to the presence of technical constraints to be considered together with the traditional requirements of system reliability.

In a deregulated environment, load-frequency control (LFC) acquires a fundamental role to enable power exchanges and to provide better conditions for the electricity trading. Since the LFC system is faced with new uncertainties in the liberalized electricity market, the modeling of these uncertainties and simulation of dynamic behavior of new structure is very important. A major challenge in new environment is to integrate computing, communication and control into appropriate levels of system operation and control. An effective power system market highly needs to an open communication infrastructure to support the increasing decentralized property of control processes.

In control systems, it is well known that time delays can degrade the system's performance and even cause system instability ${ }^{(1)-(3)}$. In light of this fact, in near future the communication delays as one of important uncertainties in LFC synthesis and analysis due to expanding physical setups, functionality, complexity of power system structure and changing the "Control area" concept is to become a significant problem ${ }^{(4)}$.

Recently, several papers are published to address the LFC modeling/synthesis in the presence of communication delays ${ }^{(5)-(7)}$. Ref. (5) focuses on the communication network requirement for a third party LFC service. A compensation method for communication time delay in the LFC systems is addressed in Ref. (6) and a control design method based on linear matrix inequalities is proposed for the LFC system with communication delays in Ref. (7).

\footnotetext{
* Department of Electrical and Computer Eng., Kumamoto University

2-39-1, Kurokami, Kumamoto 860-8555
}

This paper proposes a new control methodology to design a decentralized LFC in face of multi-delayed signals. First the PI-based LFC design is transferred to a static output feedback (SOF) control design and then to obtain the optimal PI gains, the $H_{\infty}$ control is used via a multi constraint minimization problem.

The main goal is to keep the fundamental LFC concepts and well-tested simple PI control structure to develop new LFC synthesis. In comparison of Ref. (7), simplicity of control structure, using a more complete model for delayed LFC system and no need to additional controller can be considered as advantages of the proposed methodology. This approach is applied to a 3-control area power system.

\section{Control Background}

Consider a class of time-delay systems of the form

$$
\left.\begin{array}{rl}
\dot{x}(t) & =A x(t)+B u(t)+A_{d} x(t-d)+B_{h} u(t-h)+F w(t) \\
z(t) & =C_{1} x(t) \\
y(t) & =C_{2} x(t), \quad x(t) \in \psi(t) \quad \forall t \in[-\max (d, h), 0] \\
& \ldots \ldots \ldots \ldots \ldots \ldots(1)
\end{array}\right\}
$$

Here $x \in \mathfrak{R}^{n}$ is the state, $u \in \mathfrak{R}^{n}$ is the control input, $w \in \mathfrak{R}^{n}$ is the input disturbance, $z \in \mathfrak{R}^{n}$ is the controlled output, $y \in \mathfrak{R}^{n}$ is the measured output, $C_{2} \in \mathfrak{R}^{n}$ is the constant matrix such that the pair $\left(A, C_{2}\right)$ is detectable. $d$ and $h$ represent the delay amounts in the state and the input respectively. $A \in \mathfrak{R}^{n \times n}$ and $B \in \mathfrak{R}^{n \times m}$ represent the nominal system without delay such that the pair $(A, B)$ is stabilizable. $A_{d} \in \mathfrak{R}^{n \times n}$, $B_{h} \in \mathfrak{R}^{n \times m}, F \in \mathfrak{R}^{n \times q}$ are known matrices and $\psi(t)$ is a continuous vector-valued initial function.

In this paper, the formulation is based on expressing the constraints as linear matrix inequalities (LMI) which can be easily solved using available semidefinite programming methods ${ }^{(8)(9)}$. An LMI is any constraint of the form

$$
L(x) \equiv L_{0}+\sum_{i=1}^{m} x_{i} L_{i}<0 .
$$

where $x \in \mathfrak{R}^{m}$ is the variable and $L_{i}=L_{i}^{T} \in \mathfrak{R}^{n \times n}$, 
$i=0, \cdots, m$ are given symmetric matrices. The LMI Eq. (2) is a convex constraint on $x$ and a multiple LMIs $L_{1}(x)<0, \cdots, L_{n}(x)<0$ can be expressed as the single LMI $\operatorname{diag}\left(L_{1}(x), \cdots, L_{n}(x)\right)<0$. Many control problems and design specifications have LMI formulation.

The following theorem adopts $H_{\infty}$ theory in the control synthesis for time-delay systems (using LMI description) and establishes the conditions under which the state feedback control law

$$
u(t)=K x(t)
$$

stabilizes Eq. (1) and guarantees the $H_{\infty}$ norm bound $\gamma$ of the closed-loop transfer function $T_{z w}$, namely $\left\|T_{z w}\right\|_{\infty}<\gamma ; \gamma>0$.

[Theorem 1] The state feedback controller $K$ asymptotically stabilizes the time-delay system (1) and $\left\|T_{z w}\right\|_{\infty}<\gamma$ for $d, h \geq 0$ if there exist matrices $0<P^{T}=P \in \mathfrak{R}^{n \times n}$, $0<Q_{1}^{T}=Q_{1} \in \mathfrak{R}^{n \times n}, 0<Q_{2}^{T}=Q_{2} \in \mathfrak{R}^{n \times n}$ satisfying the LMI

$$
\left[\begin{array}{ccccc}
P A_{c}+A_{c}^{T} P+Q_{1}+Q_{2} & A_{d}^{T} P & K^{T} B_{h}^{T} P & C_{1} & F^{T} P \\
P A_{d} & -Q_{1} & 0 & 0 & 0 \\
P B_{h} K & 0 & -Q_{2} & 0 & 0 \\
C_{1}^{T} & 0 & 0 & I & 0 \\
P F & 0 & 0 & 0 & -\gamma^{2} I
\end{array}\right]<0
$$

where

$$
A_{c}=A+B K
$$

[Proof] According to the Schur complement, LMI (4) is equivalent to the following matrix inequality

$$
\begin{aligned}
& P A_{c}+A_{c}^{T} P+Q_{1}+Q_{2}+P A_{d} Q_{1}^{-1} A_{d}^{T} P \\
& \quad+P B_{h} K Q_{2}^{-1} K^{T} B_{h}^{T} P+C_{1}^{T} C_{1}+\gamma^{-2} P F F^{T} P<0
\end{aligned}
$$

The sufficiency of theorem for this new inequality notation is given in Ref. (1).

\section{Proposed Control Methodology}

In this work, by augmenting the control area description to include the measured output signal and its integral, the PIbased LFC problem is transferred to a static output feedback (SOF) control problem ${ }^{(10)}$.

$$
\begin{aligned}
u(t) & =k y(t) \cdots \cdots \cdots \cdots \cdots \cdots \\
u(t) & =k_{P} A C E+k_{I} \int A C E \\
& =\left[k_{P} k_{I}\right]\left[A C E \int A C E\right]^{T}
\end{aligned}
$$

$k_{P}$ and $k_{I}$ are constant real numbers (PI parameters). $A C E$ is the area control error signal which for each control area can be expressed as a linear combination of tie-line power change and frequency deviation ( $\beta$ is the frequency bias):

$$
A C E=\beta \Delta f+\Delta P_{t i e}
$$

The main merit of this transformation is in possibility of using the well-known SOF control techniques to calculate the fixed gains, and once the SOF gain vector is obtained, the PI gains are ready in hand and no additional computation is needed.

Now, the control problem is that of designing a SOF control for the time-delay system (1) of the form of Eq. (7). $k$ is a static gain to be determined. Following theorem provides an LMI-based $H_{\infty}$ solution:

[Theorem 2] The SOF controller $k$ asymptotically stabilizes the system (1) and $\left\|T_{z w}\right\|_{\infty}<\gamma$ for $d, h \geq 0$ if there exist matrices $0<Y^{T}=Y \in \mathfrak{R}^{n \times n}, 0<Q_{t}^{T}=Q_{t} \in \mathfrak{R}^{n \times n}$ and $0<Q_{s}^{T}=Q_{s} \in \mathfrak{R}^{n \times n}$ satisfying the matrix inequality (10).

[Proof] The controller Eq. (7) can be considered as a replica of the state-feedback controller Eq. (3):

$$
u(t)=k y(t)=k C_{2} x(t)
$$

Based on theorem 1, there exists a memory-less feedback controller with constant gain

$$
K=k C_{2}
$$

such that the closed-loop system is asymptotically stable and $\left\|T_{z w}\right\|_{\infty}<\gamma$ for $d, h \geq 0$. According to Eq. (5), for the closedloop system we have

$$
A_{c}=A+B k C_{2}
$$

The stabilizing controller satisfies inequality (6). Therefore, using Eq. (13) we can write

$$
\begin{aligned}
& P\left(A+B k C_{2}\right)+\left(A+B k C_{2}\right)^{T} P+Q_{1}+Q_{2}+P A_{d} Q_{1}^{-1} A_{d}^{T} P \\
& \quad+P B_{h} k C_{2} Q_{2}^{-1}\left(k C_{2}\right)^{T} B_{h}^{T} P+C_{1}^{T} C_{1}+\gamma^{-2} P F F^{T} P<0
\end{aligned}
$$

Premultiplying and postmultiplying (14) by $P^{-1}$ and letting $P^{-1}=Y$, we get

$$
\begin{aligned}
& A Y+Y A^{T}+Y Q_{1} Y+Y Q_{2} Y+B k C_{2} Y+Y\left(k C_{2}\right)^{T} B^{T} \\
& \quad+A_{d} Q_{1}^{-1} A_{d}^{T}+B_{h} k C_{2} Q_{2}^{-1}\left(k C_{2}\right)^{T} B_{h}^{T}+Y C_{1}^{T} C_{1} Y \\
& \quad+\gamma^{-2} F F^{T}<0 \ldots \ldots \ldots \ldots \ldots \ldots \ldots \ldots \ldots \ldots \ldots \ldots \ldots \ldots \ldots
\end{aligned}
$$

Now, assuming $Y Q_{1} Y=Q_{t}, Y Q_{2} Y=Q_{s}$ and using the following inequality ${ }^{(11)}$ :

$$
W_{s}=\left[\begin{array}{cccc}
A Y+Y A^{T}+Q_{t}+Q_{s} & \left(B k C_{2}\right)^{T} & Y & Y A_{d}^{T} \\
B k C_{2} & -I_{n} & 0 & 0 \\
Y & 0 & -I_{n} & 0 \\
A_{d} Y & 0 & 0 & -Q_{t} \\
B_{h} k C_{2} Y & 0 & 0 & 0 \\
Y C_{1}^{T} & 0 & 0 & 0 \\
F & 0 & 0 & 0
\end{array}\right.
$$

$\left.\begin{array}{ccc}\left(B_{h} k C_{2} Y\right)^{T} & C_{1} Y & F^{T} \\ 0 & 0 & 0 \\ 0 & 0 & 0 \\ 0 & 0 & 0 \\ -Q_{s} & 0 & 0 \\ 0 & -I_{p} & 0 \\ 0 & 0 & -\gamma^{2} I_{q}\end{array}\right]<0$




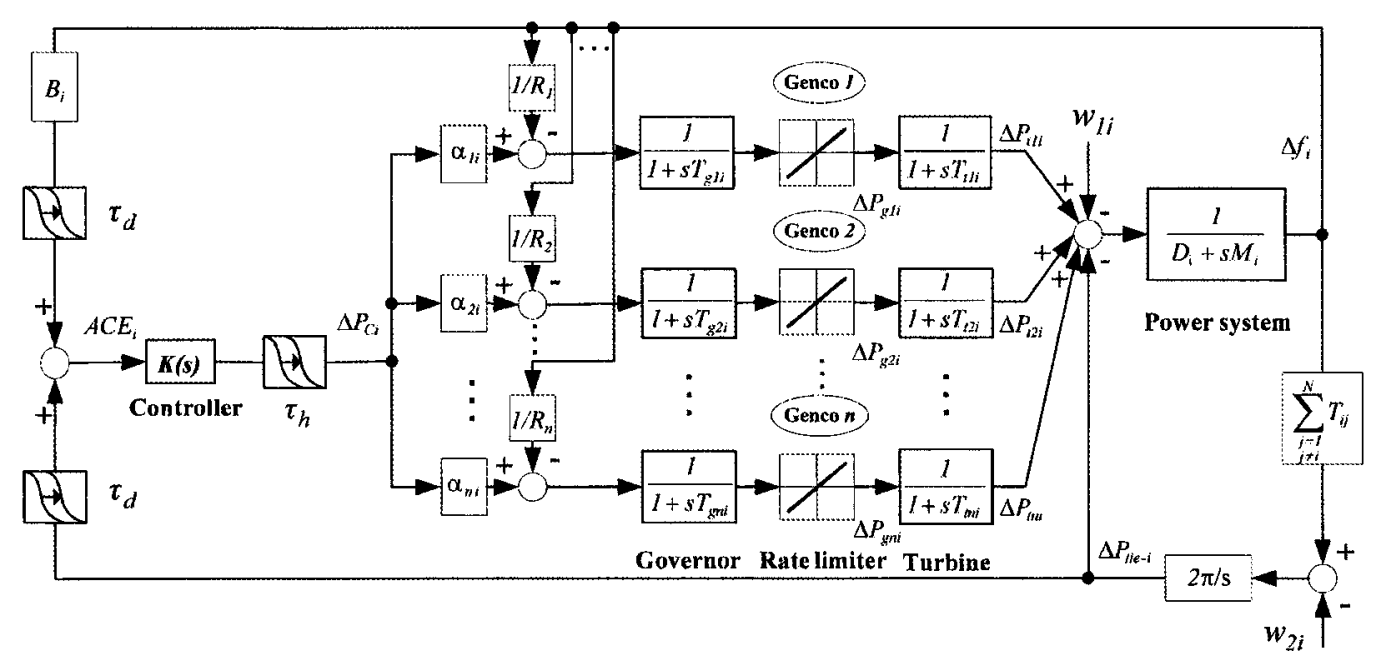

Fig. 1. LFC system with communication delays

$\forall \Omega_{1}, \Omega_{2} \in \mathfrak{R}: \Omega_{1}^{T} \Omega_{2}+\Omega_{2}^{T} \Omega_{1} \leq \alpha \Omega_{1}^{T} \Omega_{1}+\alpha^{-1} \Omega_{2}^{T} \Omega_{2}$
$\alpha>0 \ldots \ldots \ldots \ldots \ldots \ldots \ldots \ldots \ldots \ldots \ldots \ldots \ldots \ldots \ldots \ldots \ldots \ldots \ldots$

(15) can be reduced to

$$
\begin{aligned}
& {\left[A Y+Y A^{T}+Q_{t}+Q_{s}\right]+\left[B k C_{2}\left(B k C_{2}\right)^{T}+Y^{T} Y\right]} \\
& \quad+\left[A_{d} Y Q_{t}^{-1}\left(A_{d} Y\right)^{T}\right]+\left[B_{h} k C_{2} Y Q_{s}^{-1}\left(B_{h} k C_{2} Y\right)^{T}\right] \\
& \quad+Y C_{1}^{T} C_{1} Y+\gamma^{-2} F F^{T}<0 \ldots \ldots \ldots \ldots \ldots \cdots \cdots
\end{aligned}
$$

Using the Schur complement method, (17) can be arranged conveniently to yield the block form (10) as desired.

Theorem 2 shows that to determine the SOF controller $k$, one has to solve the following minimization problem:

$$
\begin{aligned}
& \min _{Q_{t}, Q_{s}, Y, k} \gamma \text { subject to }-Y<0, \\
& \quad-Q_{t}<0,-Q_{s}<0,-W_{s}<0 .
\end{aligned}
$$

The matrix inequality (10) points to an iterative approach to solve $k, Q_{t}$ and $Q_{s}$ namely, if $Y$ is fixed, then it reduces to an LMI problem in the unknown $k, Q_{t}$ and $Q_{s}$. The LMI problem is convex and can be solved efficiently using the LMI Control Toolbox ${ }^{(9)}$, if a feasible solution exists. One may use a simple optimization algorithm similar to that is given in Ref. (10).

[Remark 1] It is shown that the necessary condition for the existence of solution is that the nominal transfer function

$$
T(s)=k C_{2}[s I-A]^{-1} B
$$

is strictly positive real (SPR) ${ }^{(12)}$. To approach the solution for some positive real cases, it is possible to use a reasonable approximation to close those systems to SPR ones.

[Remark 2] It is significant to note that because of using simple constant gains, pertaining to SOF synthesis for dynamical systems in the presence of strong constraints and tight objectives are few and restrictive. Under such conditions, the minimization problem (18) may not be an approach to a strictly feasible solution.

\section{LFC with Time delays}

The traditional LFC model ${ }^{(10)(13)}$ is modified to include communication delays. These delays are considered on three communication links. The delays on the measured frequency and tie-line power flow from RTUs to control center and the produced rise/lower signal from control center to individual generation units.

The modified LFC model is given in Fig. 1. $w_{1 i}$ and $w_{2 i}$ show local load disturbance and area interface, respectively. Other parameters and variables are expressed in the Appendix. Following a load disturbance within the control area, the frequency of the area experiences a transient change and the feedback mechanism comes into play and generates appropriate control signal to make generation following the load. The balance between connected control areas is achieved by detecting the frequency and tie line power deviation via communication line to generate the ACE signal used by PI controller. The control signal is submitted to the participated Gencos via other link, based on their participation factors.

According to Eq. (1), the open-loop state space model for the LFC system of control area " $i$ " can be obtained as follows:

$$
\left.\begin{array}{l}
\dot{x}_{i}(t)=A_{i} x_{i}(t)+B_{i} u_{i}(t)+A_{d i} x_{i}(t-d)+B_{h i} u_{i}(t-h)+F_{i} w_{i}(t) \\
z_{i}(t)=C_{1 i} x_{i}(t) \\
y_{i}(t)=C_{2 i} x_{i}(t)
\end{array}\right\}
$$

Where

$$
\begin{aligned}
& x_{i}^{T}=\left[\begin{array}{lllll}
\Delta f_{i} & \Delta P_{t i e-i} & \int A C E_{i} & x_{t i} & x_{g i}
\end{array}\right] \cdots \cdots \\
& x_{t i}=\left[\begin{array}{llll}
\Delta P_{t 1 i} & \Delta P_{t 2 i} & \cdots & \Delta P_{t n i}
\end{array}\right] \\
& x_{g i}=\left[\begin{array}{llll}
\Delta P_{g 1 i} & \Delta P_{g 2 i} & \cdots & \Delta P_{g n i}
\end{array}\right] \\
& y_{i}^{T}=\left[\begin{array}{ll}
A C E_{i} & \int A C E_{i}
\end{array}\right], u_{i}=\Delta P_{C i} \\
& z_{i}^{T}=\left[\begin{array}{ll}
\eta_{1 i} \Delta f_{i} & \eta_{2 i} \int A C E_{i}
\end{array}\right] \\
& w_{i}^{T}=\left[\begin{array}{ll}
w_{1 i} & w_{2 i}
\end{array}\right]
\end{aligned}
$$

The notations, constants and matrix factors are given in Appendix.

\section{Application to a Multi Control Area}

To illustrate the effectiveness of the proposed control 
strategy, the PI-based LFC design in a 3-control area power system, shown in Fig. 2, is considered as an example. Each control area includes three generation companies (Gencos) with $9^{\text {th }}$ order.

The power system data and parameters are considered as the same as in Ref.(13). It is assumed that the maximum frequency/tie-line and control signal delays for each control area are as follows:

$$
d_{i}=1.5 \mathrm{~s}, h_{i}=2 \mathrm{~s} ; i=1,2,3
$$

Based on the given simple stability condition in Ref. (14), the open loop system (20) with real matrices is stable if

$$
\mu\left(A_{i}\right)+\left\|A_{d i}\right\|<0
$$

where

$$
\mu\left(A_{i}\right)=\frac{1}{2} \max _{j} \lambda_{j}\left(A_{i}^{T}+A_{i}\right)
$$

Here, $\lambda_{j}$ denotes the $j$ th eigenvalue of $\left(A_{i}^{T}+A_{i}\right)$. Using above stability rule, we note that for the example at hand, the control areas are unstable:

$$
\begin{aligned}
& \mu\left(A_{1}\right)+\left\|A_{d 1}\right\|=10.4736>0 \\
& \mu\left(A_{2}\right)+\left\|A_{d 2}\right\|=12.2615>0 \\
& \mu\left(A_{3}\right)+\left\|A_{d 3}\right\|=10.2285>0
\end{aligned}
$$

According to the synthesis methodology described in section 3 , a set of three decentralized robust PI controllers are obtained as shown in Table 1.

Based on theorem 2, since a solution for the time-delayed

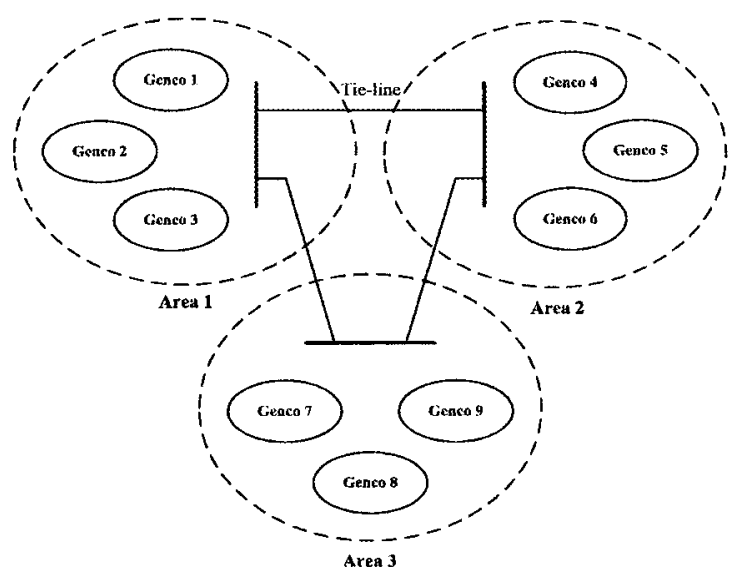

Fig. 2. Three control area power system

Table 1. PI control parameters using the proposed control design

\begin{tabular}{|c|c|c|c|}
\hline Parameters & Area 1 & Area 2 & Area 3 \\
\hline$k_{P i}$ & 0.0250 & 0.0396 & 0.0308 \\
\hline$k_{I i}$ & -0.1888 & -0.2520 & -0.2753 \\
\hline
\end{tabular}

Table 2. PI control parameters using the nominal control design

\begin{tabular}{|c|c|c|c|}
\hline Parameters & Area 1 & Area 2 & Area 3 \\
\hline$k_{P i}$ & 0.1473 & -0.0571 & 0.0023 \\
\hline$k_{l i}$ & -0.3464 & -0.5650 & -0.5459 \\
\hline
\end{tabular}

LFC problem will be obtained trough the minimizing the guaranteed $H_{\infty}$ performance index $\gamma$ (as a valid performance measure) subject to the given constraints in (18), the designed PI controllers satisfy the robustness of the closedloop system. In other words, the basis of designing the SOF controllers Eq. (7) is to simultaneously stabilize Eq. (20) and guarantee the $H_{\infty}$-norm bound $\gamma$ of the closed-loop transfer
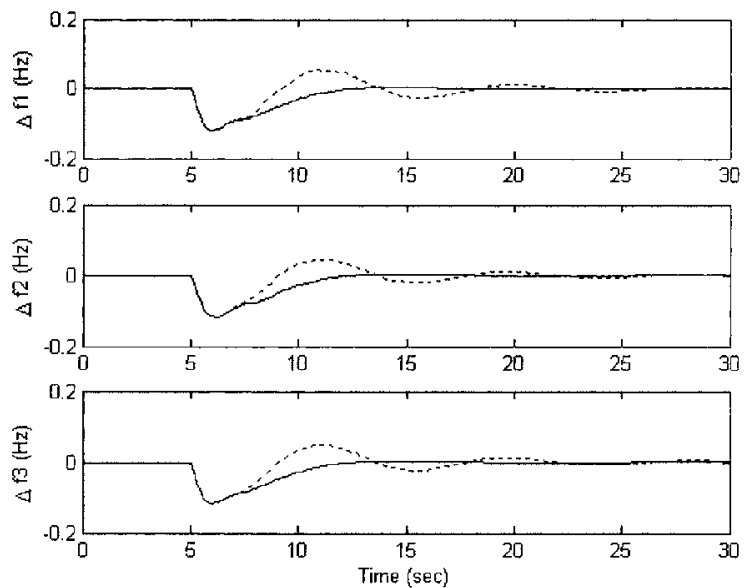

(a)
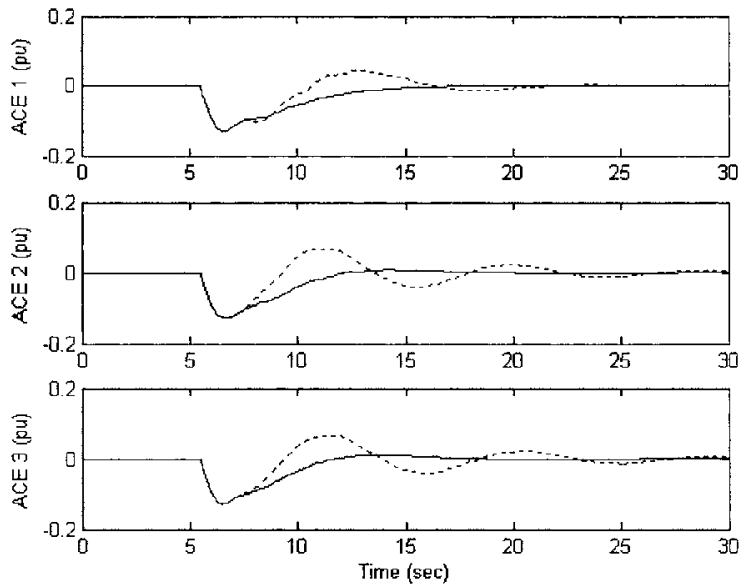

(b)
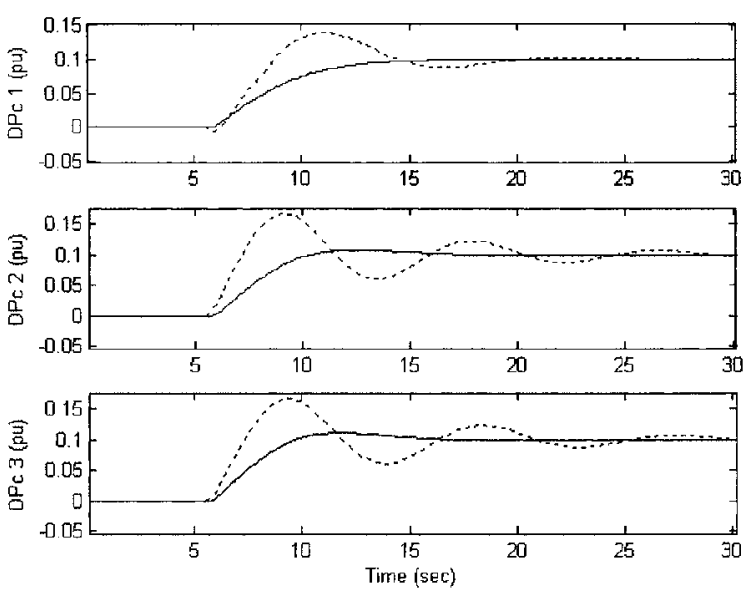

(c)

Fig. 3. System response for $d_{i}=0.5 \mathrm{~s}, h_{i}=1 \mathrm{~s}$. Solid (proposed design), dotted (conventional design): (a) frequency deviation, (b) ACE and (c) control effort 

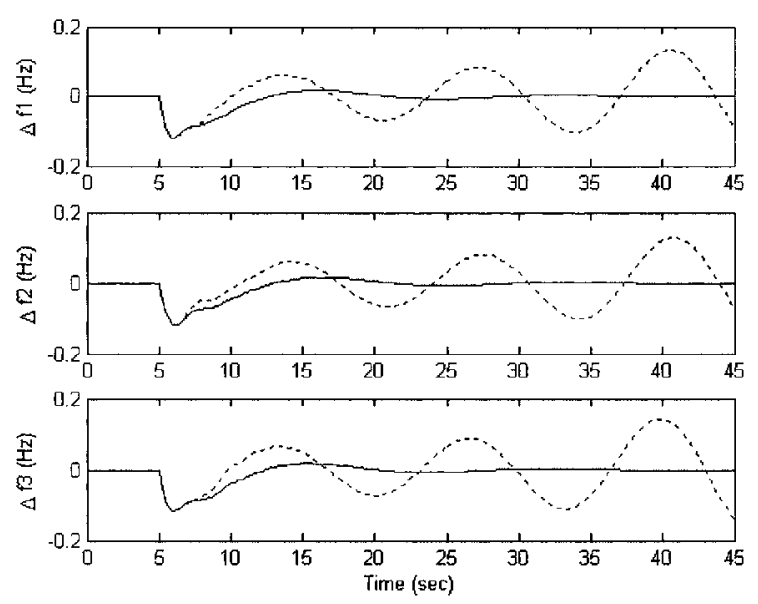

Fig. 4. System response for $d_{1}=h_{1}=1 \mathrm{~s}, d_{2}=h_{2}=$ $0.5 \mathrm{~s}, d_{3}=h_{3}=1.5 \mathrm{~s}$; Solid (proposed design), dotted (conventional design)

function $T_{z w}$, namely,

$$
\left\|T_{z w}\right\|_{\infty}<\gamma ; \gamma>0 \text {. }
$$

For comparisons, a robust PI controller is designed for nominal system (without delays) of each control area using the $H_{\infty}$-SOF control technique ${ }^{(10)}$. The resulted parameters are given in Table 2.

\section{Simulation Results}

In order to demonstrate the effectiveness of the proposed strategy, some simulations were carried out. Fig. 3 shows the closed-loop response (frequency deviation, area control error and control action signals) in presence of delays

$$
d_{i}=0.5 \mathrm{~s}, h_{i}=1 \mathrm{~s} ; i=1,2,3
$$

following a 0.1 pu step load disturbance at $5 \mathrm{~s}$ in each control area. Both types of designed controllers act to return the frequency and ACE signals to scheduled values, however the applied delays degrade the system performance for nominal (delay-less) system based $H_{\infty}$ control design.

Increasing the delays will degrade the conventional LFC system performance seriously. Fig. 4 shows the frequency deviation for control areas in face of $1 \mathrm{~s}, 0.5 \mathrm{~s}$ and $1.5 \mathrm{~s}$ delays in communication channels of area 1, area 2 and area 3 respectively. It shows that the conventional $H_{\infty}$ controllers are not capable to hold the stability of closed-loop system.

\section{Conclusion}

An $H_{\infty}$-SOF control design is proposed to synthesis of PI based LFC system with multiple communication delays. The proposed method was applied to a 3-control area power system and the results are compared with delay less system based $H_{\infty}$ control design. The simulations demonstrate the viability of the proposed method.

\section{Acknowledgment}

This work is supported by Japan Society for the Promotion of Science (JSPS) under grant P04346. Special thanks go to Prof. Magdi S. Mahmoud from United Arab Emirates University for his helpful suggestions.

(Manuscript received Feb. 28, 2005,

revised May 20, 2005)

\section{References}

( 1 ) M.S. Mahmoud: Robust control and filtering for time-delay systems, Marcel Dekker Inc., New York (2000)

( 2 ) J. Aweya, D.Y. Montuno, and M. ouellette: "Effects of control loop delay on the stability of a rate control algorithm", Int. J. Commun. Syst., Vol.17, pp.833-850 (2004)

( 3 ) J.K. Yook, D.M. Tilbury, and N.R. Soparkar: "A design methodology for distributed control systems to optimize performance in the presence of time delays", Int. J. Control, Vol.74, No.1, pp.58-76 (2001)

( 4 ) H. Bevrani, Y. Mitani, K. Tsuji, and H. Bevrani: "Bilateral-based robust loadfrequency control", Energy Conversion \& Management, Vol.46, pp.1129$1146(2005)$

( 5 ) S. Bhowmik, K. Tomosovic, and A. Bose: "Communication models for third party load frequency control”, IEEE Trans. Power Syst., Vol.19, No.1, pp.543-548 (2004)

( 6 ) T. Hiyama, T. Nagata, and T. Funabashi: "Multi-agent based automatic generation control of isolated stand alone power system", Proc. of Int. Conf. on Power System Technology, Vol.1, pp.139-143 (2004)

( 7 ) X. Yu and K. Tomosovic: "Application of linear matrix inequalities for load frequency control with communication delays", IEEE Trans. Power Syst., Vol.19, No.3, pp.1508-1515 (2004)

( 8 ) S. Boyd, L. El. Ghaoui, E. Feron, and V. Balakrishnan: "Linear Matrix Inequalities in Systems and Control Theory", SIAM Books, Vol.15, Philadelphia (1994)

( 9 ) P. Gahinet, A. Nemirovski, A.J. Laub, and M. Chilali: LMI Control Toolbox, The MathWorks, Inc. (1995)

(10) H. Bevrani, Y. Mitani, and K. Tsuji: "Robust decentralized load-frequency control using an iterative linear matrix inequalities algorithm", IEE Proc. Gener. Transm. Distrib., Vol.151, No.3, pp.347-354 (2004)

(11) M.S. Mahmoud and M. Zribi: "Guaranteed cost observer-based control of uncertain time-lag systems”, Comput. Elect. Eng., Vol.29, pp.193-212 (2003)

(12) K.S. Narendra and A.M. Annaswamy: Stable adaptive systems, PrenticeHall, Englewood Cliffs, New Jersey (1989)

(13) D. Rerkpreedapong, A. Hasanovic, and A. Feliachi: "Robust load frequency control using genetic algorithms and linear matrix inequalities", IEEE Trans. Power Syst., Vol.18, No.2, pp.855-861 (2003)

(14) T. Mori and H. Kokame: "Stability of $\dot{x}(t)=A x(t)+B x(t-\tau)$ ", IEEE Trans. Automatic Control, Vol.34, pp.460-462 (1989)

\section{Appendix}

In Eq. (20), the following matrices are defined:

$$
\begin{aligned}
& A_{i}=\left[\begin{array}{lll}
A_{i 11} & A_{i 12} & A_{i 13} \\
A_{i 21} & A_{i 22} & A_{i 23} \\
A_{i 31} & A_{i 32} & A_{i 33}
\end{array}\right], \quad A_{d i}=\left[\begin{array}{ccc}
A_{i 11} & 0_{3 \times n} & 0_{3 \times n} \\
0_{n \times 3} & 0_{n \times n} & 0_{n \times n} \\
A_{i 31} & 0_{n \times n} & 0_{n \times n}
\end{array}\right] \\
& B_{i}=\left[\begin{array}{l}
B_{i 1} \\
B_{i 2} \\
B_{i 3}
\end{array}\right], \quad B_{h i}=B_{i}, \quad F_{i}=\left[\begin{array}{c}
F_{i 1} \\
F_{i 2} \\
F_{i 3}
\end{array}\right] \\
& C_{1 i}=\left[\begin{array}{lll}
c_{1 i} & 0_{2 \times n} & 0_{2 \times n}
\end{array}\right], \quad c_{1 i}=\left[\begin{array}{cc}
\eta_{1 i} & 0 \\
0 & \eta_{2 i}
\end{array}\right] \\
& C_{2 i}=\left[\begin{array}{lll}
c_{2 i} & 0_{2 \times n} & 0_{2 \times n}
\end{array}\right], \quad c_{2 i}=\left[\begin{array}{ccc}
B_{i} & 1 & 0 \\
0 & 0 & 1
\end{array}\right] \\
& A_{i 11}=\left[\begin{array}{ccc}
-D_{i} / M_{i} & -1 / M_{i} & 0 \\
2 \pi \sum_{\substack{j=1 \\
j \neq i \\
\beta_{i}}}^{N} T_{i j} & 0 & 0 \\
\beta_{i} & 1 & 0
\end{array}\right] \\
& A_{i 12}=\left[\begin{array}{ccc}
1 / M_{i} & \cdots & 1 / M_{i} \\
0 & \cdots & 0 \\
0 & \cdots & 0
\end{array}\right]_{3 \times n} \\
& A_{i 22}=-A_{i 23}=\operatorname{diag}\left[-1 / T_{t 1 i}-1 / T_{t 2 i} \cdots-1 / T_{t n i}\right] \\
& A_{i 33}=\operatorname{diag}\left[-1 / T_{g 1 i}-1 / T_{g 2 i} \cdots-1 / T_{g n i}\right]
\end{aligned}
$$




$$
\begin{aligned}
& A_{i 31}=\left[\begin{array}{ccc}
-1 /\left(T_{g 1 i} R_{1 i}\right) & 0 & 0 \\
\vdots & \vdots & \vdots \\
-1 /\left(T_{g n i} R_{n i}\right) & 0 & 0
\end{array}\right] \\
& A_{i 13}=A_{i 21}^{T}=0_{3 \times n} \\
& A_{i 32}=0_{n \times n} \\
& F_{i 1}=\left[\begin{array}{cc}
-1 / M_{i} & 0 \\
0 & -2 \pi \\
0 & 0
\end{array}\right] \\
& F_{i 2}=F_{i 3}=0_{n \times 2} \\
& B_{i 1}=0_{3 \times 1}, B_{i 2}=0_{n \times 1} \\
& B_{i 3}^{T}=\left[\begin{array}{llll}
\alpha_{1 i} / T_{g 1 i} & \alpha_{2 i} / T_{g 2 i} & \cdots & \alpha_{n i} / T_{g n i}
\end{array}\right] \\
& \eta_{1}=0.5, \eta_{2}=1
\end{aligned}
$$

where

$\Delta f_{i}$ : frequency deviation,

$\Delta P_{g i}:$ governor valve position,

$\Delta P_{c i}:$ governor load setpoint,

$\Delta P_{t i}$ : turbine power,

$\Delta P_{\text {tie- } i}:$ net tie-line power flow,

$M_{i}$ : equivalent inertia constant,

$D_{i}$ : equivalent damping coefficient,

$T_{g i}$ : governor time constant,

$T_{t i}$ : turbine time constant,
$T_{i j}$ : tie-line synchronizing coefficient for area $i \& j$,

$\beta_{i}$ : frequency bias,

$R_{k}$ : drooping characteristic,

$A C E_{i}$ : area control error (ACE),

$\alpha_{k i}:$ ACE participation factors.

Hassan Bevrani (Member) received his B.S., M.S. and Ph.D. degrees in Electrical Engineering from Mashad University (Iran, 1991), K. N. Toosi University of Technology (Iran, 1997) and Osaka University (Japan, 2004), respectively. Currently, he is an assistant professor of Kurdistan University (Iran) and a postdoctoral fellow in Kumamoto University (Japan). His special fields of interest include robust load-frequency control and robust/intelligent control applications in Power system and Power electronic industry. $\mathrm{He}$ is a member of the Institute of Electrical Engineers of Japan, IEEE and IEE.

Takashi Hiyama (Member) was born on March 14, 1947. He re-

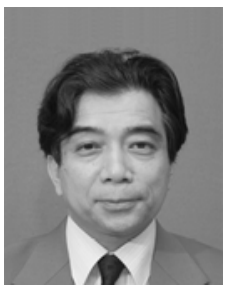
ceived his B.E., M.S., and Ph.D. degrees all in Electrical Engineering from Kyoto University, in 1969, 1971, and 1980, respectively. Since 1989, he has been a Professor at the Department of the Electrical and Computer Engineering, Kumamoto University. His current interests include the application of intelligent systems to power system operation, management, and control. He is a senior member of IEEE, a member of Japan Solar Energy Society, and a member of SICE. 